\title{
Predicting the pathogenesis of influenza from genomic response: a step toward early diagnosis
}

\author{
Eric Bortz and Adolfo García-Sastre*
}

\begin{abstract}
Infection with influenza virus does not always lead to symptomatic illness, but it is not currently possible to predict who will be severely affected and who will have mild or no symptoms. Gene expression profiling of biofluids might unlock the complex dynamics of response to acute respiratory virus infections such as influenza. A recent article by Alfred Hero and colleagues used transcriptional microarray analyses to follow the response to symptomatic and asymptomatic influenza infection over time, and revealed a role for type I IFN (IFN $\beta$ and IFNa) signaling and the NLRP3 inflammasome in determining the outcome in human infections.
\end{abstract}

Influenza viruses are RNA viruses and are a common cause of respiratory infection, with seasonal type A and B influenza viruses reaching peak prevalence during the winter months. As the 2009 H1N1 pandemic influenza and the sporadic human deaths from H5N1 highly pathogenic avian influenza illustrate, the risk of novel pathogenic strains emerging from animal reservoirs is ever present. Understanding of the molecular bases of viral pathogenesis, human susceptibility and immune responses is crucial for the development of new vaccines, targeted diagnostics and antiviral drugs.

Human exposure to influenza viruses can lead to a wide variety of pathological outcomes. Whereas some people succumb to symptomatic (Sx) infection, others apparently show few symptoms. Severe influenza A virus infections can result in pneumonia and immunopathology characterized by high oxidative stress, high levels of pro-inflammatory chemokines and cytokines (hypercytokinemia), and acute respiratory distress syndrome [1,2]. The influenza virus is cleared mainly through the generation of cytotoxic $\mathrm{T}$ lymphocytes (CTLs) and neutralizing

*Correspondence: adolfo.garcia-sastre@mssm.edu

Department of Microbiology, Mount Sinai School of Medicine, One Gustave L Levy Place, New York, NY 10029, USA antibodies, and future infections are prevented by adaptive immune responses that are capable of targeting antigenically similar strains. Neutralizing antibodies, which are generated during natural infection and by currently approved influenza vaccines, primarily target surface glycoproteins, hemagglutinin and neuraminidase and are strongly associated with protection. However, the first response to influenza virus infection is mediated by the innate immune system.

The early host innate immune response is triggered by recognition of the viral RNA by pathogen-recognition receptors (PRRs; such as Rig-I, TLR3/7 and NLRP3). Responding primarily in antigen-presenting dendritic cells resident in the respiratory tract, Rig-I and TLR3/7 induce type I IFN (IFN $\beta$ and IFN $\alpha$ ). Induction of IFN $\beta / \alpha$ leads to induction of antiviral, interferon-stimulated genes and cytokine/chemokine signals that recruit innate immune effector cells and adaptive immune mechanisms $[3,4]$. In influenza virus infection, oxidative stress also activates the NLRP3 inflammasome, which is an oligomeric complex of NLRP3 that senses cellular 'danger signals' and possibly viral RNA [5]. NLRP3 contains a caspase activation and recruitment domain that activates caspase 1, which in turn leads to the proteolytic processing and release of pro-inflammatory cytokines IL1 $\beta$ and IL18 [6]. The NLRP3 inflammasome facilitates influenza virus clearance by mediating recruitment of innate immune cells to the lung, and is required for lung repair and survival in mice [5,7].

Microarray analyses of host responses to experimental influenza virus infection in animal models have yielded an unprecedented look into the complex molecular dynamics regulating viral pathogenesis, innate and adaptive immune responses, and inflammation. However, the transcriptional dynamics of host responses to influenza virus infections in humans have been less well studied. To address this question, Huang et al. [8] undertook a novel set of clinical genomics experiments, reported in PLoS Genetics, which we discuss below.

\section{Dynamic response to infection}

Huang et al. [8] recruited human volunteers and infected them with the human seasonal influenza strain A/ 


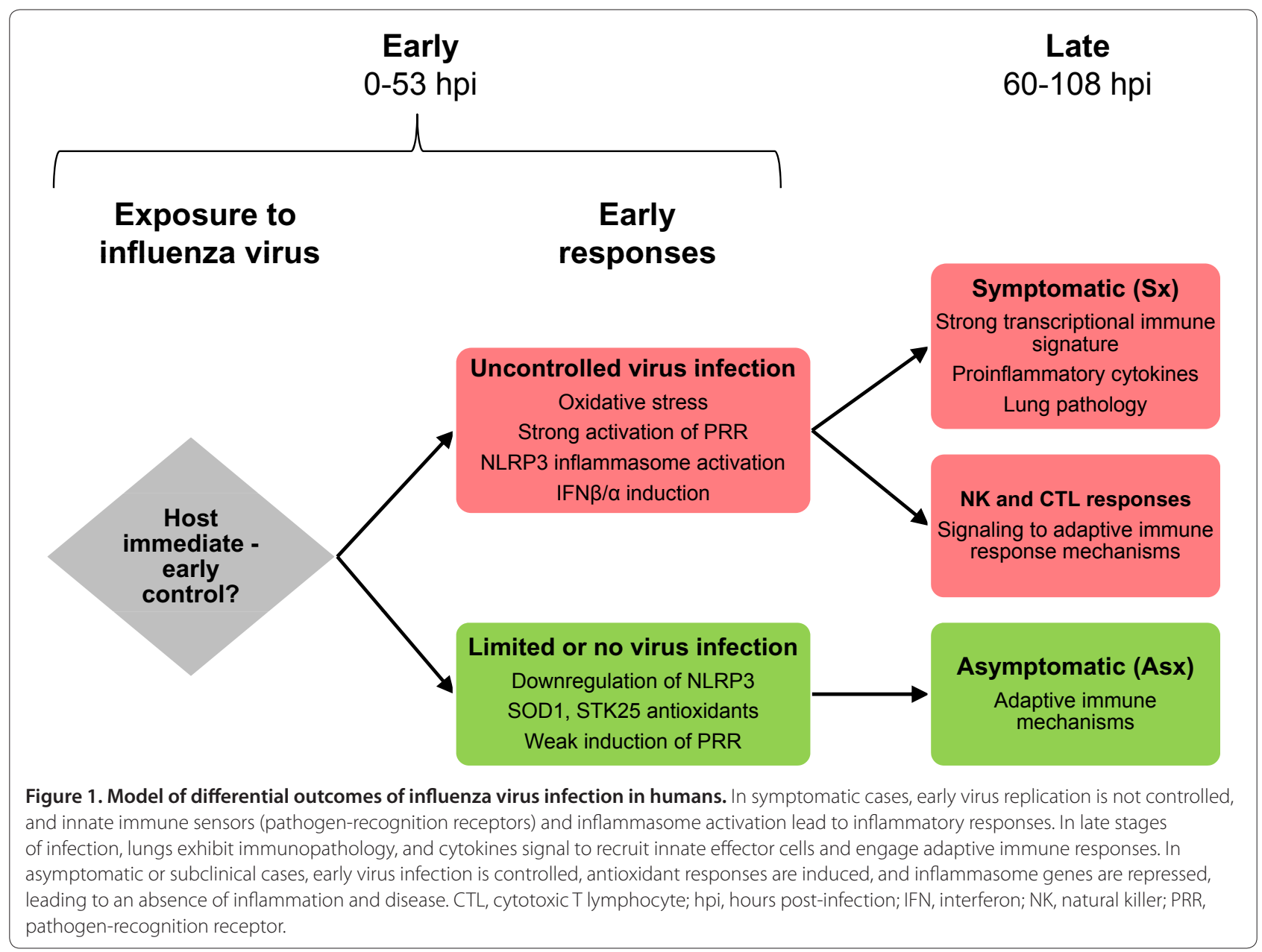

Wyoming/67/2005 (H3N2). The authors mapped the peripheral blood cell transcriptional responses using microarrays before and during the course of infection $(0$ to $108 \mathrm{~h}$ post-infection). Importantly, all volunteers were immunologically naïve for H3N2-specific antibodies and had not been vaccinated for at least 3 years.

Significant differences in transcriptional profiles between $\mathrm{Sx}$ and subclinical/asymptomatic (Asx) infection were found. Applying rigorous statistical methods, including unsupervised Bayesian analysis and clinically informed pathway enrichment, Huang et al. mapped the temporal dynamics of clusters of genes and corresponding cellular pathways. In Sx subjects, influenza virus significantly induced antiviral, inflammatory and immune response gene clusters over the course of an infection [8]. They also detected distinct profiles in Asx patients.

\section{The inflammasome is a key to host response}

There is substantial evidence that influenza virus infection causes the induction of specific innate immune, inflammatory and oxidative stress pathways in lung tissue and peripheral blood cells in humans and animal models
$[1,9,10]$. Induction of the NLRP3 inflammasome pathways in particular correlate with the onset of symptoms, and are highly upregulated in lung tissue in lethal 1918 influenza virus infection in macaques [1]. Similarly, Huang et al. found that symptomatic influenza virus infection induces a gene signature in peripheral blood cells that is enriched in pathogen recognition receptor, type I IFN, inflammasome and pro-inflammatory cytokine response genes [8]. While induction of these pathways was not unexpected, the novelty of this study lies in comparison with Asx subjects.

In contrast to Sx infection, suppression of the inflammasome by limiting oxidative stress may dictate a successful early response to infection, leading to Asx exposure. Huang et al. present substantial evidence that the Asx group showed 'subclinical' influenza virus infection (50\% shed virus, $25 \%$ showed seroconversion, and all showed similar expression changes in 3,000 genes) [8]. This suggests that the Asx phenotype was evidence of host limitation of influenza virus infection rather than a failed inoculation. Asx subjects showed differential upregulation of antioxidant genes (SOD1 and STK25) and 
suppressor of cytokine genes (SOCS2 and SOCS5), and suppression of NLRP3. Taking into account that the peripheral blood cells from which the gene expression profile were taken are at a distance from the site of influenza virus replication (the upper respiratory tract and lungs), the nature of the Asx phenotype is suggestive of an 'immediate-early' decision point upon influenza virus infection of the respiratory tract (Figure 1). Control of oxidative stress and suppression of the NLRP3 inflammasome may directly limit viral replication, or correlate with induction of a potent antiviral response that targets virus-infected cells before enough viral RNA is produced and recognized by PRR to lead to widespread induction of innate immune pathways such as IFN $\beta / \alpha$.

However, there is a confounding possibility. Asx subjects may have been able to mount a CTL-mediated immune response upon infection; it is possible that Asx subjects might only have been immunologically naïve in terms of H3N2 antibody titers. Protein synthesis machinery was strongly induced in peripheral blood cell RNA of Asx subjects, and there were significant increases in peripheral leukocyte counts [8]. Despite this, influenzaspecific CTL activity was not measured, so it is unknown whether the Asx phenotype was dependent on the presence of circulating, respiratory-tract-resident or lung-resident T-cell memory targeting conserved crossreactive influenza virus epitopes. Indeed, CTLs raised (in mice) against $\mathrm{H} 3 \mathrm{~N} 2$ virus are capable of recognizing epitopes in internal proteins (nucleoprotein and polymerase) of a divergent avian $\mathrm{H} 5 \mathrm{~N} 1$ virus, and contributing to a cross-protective, heterosubtypic response [11].

\section{Genomics leads the way}

Besides investigating the nature of the immediate-early repression of virus replication observed in Asx subjects, future genomics studies in human and animal models of influenza virus infections can apply similarly innovative approaches to genomics and clinical/physiological data analysis [8] to yield deeper insight into differences in gene expression signatures in distinct leukocyte subsets in peripheral blood. How do gene expression patterns in peripheral blood cell subsets compare with those in cell populations (epithelial, endothelial, lymphocytic, dendritic cells and macrophages) in the respiratory tract [9]? From a practical view, it is likely that biomarkers that differentiate early from late symptomatic influenza virus infection could be obtained by analyzing peripheral blood [8]. Advancing genomics-based diagnostic methods may not only contribute to understanding the molecular events leading to symptomatic influenza in human patients, but may also aid the development of point-of-care diagnostics for patients and their close contacts for the early initiation of antiviral treatment.

\section{Abbreviations}

AsX, subclinical/asymptomatic; CTL, cytotoxic T lymphocyte; IFN, interferon; IL, interleukin; PRR, pathogen-recognition receptor; Sx, symptomatic.

\section{Competing interests}

The authors declare that they have no competing interests.

\section{Acknowledgement}

The authors thank Mirco Schmolke, PhD, for helpful discussions.

\section{Published: 24 October 2011}

\section{References}

1. Cilloniz C, Shinya K, Peng X, Korth MJ, Proll SC, Aicher LD, Carter VS, Chang JH, Kobasa D, Feldmann F, Strong JE, Feldmann H, Kawaoka Y, Katze MG: Lethal influenza virus infection in macaques is associated with early dysregulation of inflammatory related genes. PLoS Pathog 2009, 5:e1000604.

2. de Jong MD, Simmons CP, Thanh TT, Hien VM, Smith GJ, Chau TN, Hoang DM, Chau NV, Khanh TH, Dong VC, Qui PT, Cam BV, Ha do Q, Guan Y, Peiris JS, Chinh NT, Hien TT, Farrar J: Fatal outcome of human influenza A (H5N1) is associated with high viral load and hypercytokinemia. Nat Med 2006, 12:1203-1207.

3. Hui KP, Lee SM, Cheung CY, Ng IH, Poon LL, Guan Y, Ip NY, Lau AS, Peiris JS: Induction of proinflammatory cytokines in primary human macrophages by influenza $A$ virus ( $\mathrm{H} 5 \mathrm{~N} 1$ ) is selectively regulated by IFN regulatory factor 3 and p38 MAPK. J Immunol 2009, 182:1088-1098.

4. Ehrhardt C, Seyer R, Hrincius ER, EierhoffT, WolffT, Ludwig S: Interplay between influenza $A$ virus and the innate immune signaling. Microbes Infect 2009, 12:81-87.

5. Allen IC, Scull MA, Moore CB, Holl EK, McElvania-TeKippe E, Taxman DJ, Guthrie EH, Pickles RJ, Ting JP: The NLRP3 inflammasome mediates in vivo innate immunity to influenza A virus through recognition of viral RNA. Immunity 2009, 30:556-565.

6. Schroder K, Tschopp J: The inflammasomes. Cell 2010, 140:821-832.

7. Thomas PG, Dash P, Aldridge JR, Jr, Ellebedy AH, Reynolds C, Funk AJ, Martin WJ, Lamkanfi M, Webby RJ, Boyd KL, Doherty PC, Kanneganti TD: The intracellular sensor NLRP3 mediates key innate and healing responses to influenza A virus via the regulation of caspase-1. Immunity 2009, 30:566-575

8. Huang Y, Zaas AK, Rao A, Dobigeon N, Woolf PJ, Veldman T, Oien NC, McClain MT, Varkey JB, Nicholson B, Carin L, Kingsmore S, Woods CW, Ginsburg GS, Hero AO 3rd: Temporal dynamics of host molecular responses differentiate symptomatic and asymptomatic influenza a infection. PLoS Genet 2011, 7:e1002234

9. Baas T, Baskin CR, Diamond DL, Garcia-Sastre A, Bielefeldt-Ohmann H, Tumpey TM, Thomas MJ, Carter VS, Teal TH, Van Hoeven N, Proll S, Jacobs JM, Caldwell ZR, Gritsenko MA, Hukkanen RR, Camp DG 2nd, Smith RD, Katze MG: Integrated molecular signature of disease: analysis of influenza virusinfected macaques through functional genomics and proteomics. J Virol 2006, 80:10813-10828.

10. Zaas AK, Chen M, Varkey J, Veldman T, Hero AO 3rd, Lucas J, Huang Y, Turner R, Gilbert A, Lambkin-Williams R, Øien NC, Nicholson B, Kingsmore S, Carin L, Woods CW, Ginsburg GS: Gene expression signatures diagnose influenza and other symptomatic respiratory viral infections in humans. Cell Host Microbe 2009, 6:207-217.

11. Kreijtz JH, Bodewes R, van den Brand JM, de Mutsert $G$, Baas $C$, van Amerongen G, Fouchier RA, Osterhaus AD, Rimmelzwaan GF: Infection of mice with a human influenza $\mathrm{A} / \mathrm{H} 3 \mathrm{~N} 2$ virus induces protective immunity against lethal infection with influenza A/H5N1 virus. Vaccine 2009, 27:4983-4989.

doi:10.1186/gm283

Cite this article as: Bortz E, García-Sastre A: Predicting the pathogenesis of influenza from genomic response: a step toward early diagnosis. Genome Medicine 2011, 3:67. 